\title{
How do Front-Of-Pack nutrition and health labels affect expected liking, and portion selection of cheddar cheeses?
}

Dairy products contribute significantly to saturated fat (SFA) intake, providing $20 \%$ of population intakes, on average ${ }^{(1)}$. Due to the association with blood cholesterol level, SFA are regarded as a risk factor for CVD. Growing evidence suggests that the food source of the SFA moderates this risk, and cheese consumption in particular is associated with neutral or beneficial outcomes in prospective intake studies ${ }^{(2)}$. However, many consumers remain wary of cheese and may avoid it or choose reduced-fat alternatives. Alternatives are sometimes considered to compromise on taste and other sensory characteristics. Since Front-of-pack (FOP) labels may impact consumer perceptions of a food, and subsequently, the chosen portion ${ }^{(3,4)}$, the aim of this study was to examine the impact of nutrition and health labelling on portion selection, expected liking, and energy estimation of four different cheeses.

149 participants $(n=45$ Males) completed an online survey with a portion selection task using Qualtrics, based on adaptations of previous methods ${ }^{(3,4)}$. Participants were presented with four 'mock' labels; (A) Full Fat cheese (B) Reduced fat, (C) Full fat 'Heart Healthy' and (D) Reduced Fat 'Heart Healthy'. Participants were asked to select one portion from 100 cheese pictures to eat for lunch on a slice of toast. The pictures varied from $1.2 \mathrm{~g}$ to $120 \mathrm{~g}$. Participants were asked to estimate the energy content of the picture chosen. Differences in sensory characteristics, energy and portion size for the cheeses were tested via ANOVA and proportions of those underand overestimating the energy content were tested with Chi-Square. Statistical analyses were conducted via SPSS v24 (IBM).

Nutrition and health claims affected expected liking, which was different for all cheeses $(\mathrm{p}<0.001)$, and highest for the full fat cheese. Males and females differed in their response to health claim and portion selection; Females consistently selected smaller portions for light cheese $(p<0.05)$ but not for the remaining cheeses. This was not seen in males. Both males and females tended to overestimate the energy content. Females were more likely to do so, with $5 \%$ of females underestimating the energy in the full fat cheese compared to $23 \%$ of males $(\mathrm{p}<0.01)$. The level of overestimation was lower in the cheeses with a health and/or a nutrition claim.

This preliminary analysis demonstrates that FOP labelling has a significant impact on the expected liking and portion selection of a product, and that this impact is different for males and females. Further research will explore the interacting factors that may impact sensory expectations and portion selection.

1. Feeney E et al. (2016) Br J Nutr, 115, 709-17.

2. Chen et al. (2016) Eur J Nutr. 56, 2565-2575.

3. McCrickerd K et al. (2020) Food Quality and Preference 79, 103766

4. Wilkinson L et al. (2012) Appetite 59, 933-938. 